\title{
Comparison of two different modes for noninvasive mechanical ventilation in chronic respiratory failure: volume versus pressure controlled device
}

\author{
B. Schönhofer*, M. Sonneborn*, P. Haidl*, H. Böhrer**, D. Köhler*
}

Comparison of two different modes for noninvasive mechanical ventilation in chronic respiratory failure: volume versus pressure controlled device. B. Schönhofer, $M$. Sonneborn, P. Haidl, H. Böhrer, D. Köhler. @ERS Journals Ltd 1997.

ABSTRACT: The most commonly used mode of noninvasive mechanical ventilation (NMV) is volume-controlled intermittent positive pressure ventilation (IPPV). Pressure support ventilation has recently become increasingly popular, but its merits have not been clearly defined.

In an open, nonrandomized follow-up study, we evaluated two modes of NMV, volume-controlled (IPPV) and pressure-controlled ventilation (PCV) over 6 months in 30 consecutive patients ( 24 males and 6 females, aged $49 \pm 19$ yrs) with chronic respiratory failure (CRF). The baseline assessments comprised both physiological and subjective data.

In all cases, nasal IPPV was initially administered for 1 month, followed by a second month of nasal PCV. Thereafter, responders or nonresponders to PCV were defined according to the patients' subjective symptom score and/or the recurrence of hypercapnia. During the IPPV phase, in all but two patients the subjective and objective parameters improved significantly. During the following 1 month PCV phase, stabilization was maintained in 18 patients ("responders"), while 10 patients were defined as "nonresponders". In nonresponders, hypercapnia increased (arterial carbon dioxide tension $\left(\mathrm{Pa}_{\mathrm{a}} \mathrm{CO}_{2}\right): 5.7 \pm 0.4$ to $6.6 \pm 0.5 \mathrm{kPa}$; $\mathbf{p}<0.05$ ) and symptom scores decreased. Compared with responders, nonresponders had a lower mean nocturnal arterial oxygen saturation $\left(S_{\mathrm{a}}, \mathrm{O}_{2}\right)(\mathrm{p}<0.05)$ and a higher daytime $\mathrm{Pa}_{\mathrm{a}} \mathrm{CO}_{2}(\mathrm{p}<0.05)$ at baseline.

We conclude that the majority of patients suffering from chronic respiratory failure who are initially satisfactorily ventilated with intermittent positive pressure ventilation may also be adequately maintained with pressure-controlled ventilation. However, there is a subgroup with more severe chronic respiratory failure at baseline, in whom pressure-controlled ventilation is inadequate. After 4 weeks of treatment with pressure-controlled ventilation, the subjective scores and the arterial carbon dioxide tension values reliably distinguished between long-term responders and nonresponders to pressure-controlled ventilation.

Eur Respir J., 1997; 10: 184-191.

\begin{abstract}
*Krankenhaus Kloster Grafschaft, Zentrum für Pneumologie und Allergologie, Schmallenberg-Grafschaft, Germany. **Klinik für Anaesthesiologie, RuprechtKarls-Universität Heidelberg, Germany.

Correspondence: B. Schönhofer Krankenhaus Kloster Grafschaft Zentrum für Pneumologie und Allergologie D-57392 Schmallenberg-Grafschaft Germany
\end{abstract}

Keywords: Chronic respiratory failure noninvasive ventilation pressure-controlled ventilation volume-controlled ventilation

Received: April 121995 Accepted after revision June 261996
Chronic respiratory failure (CRF) is caused by a decrease in the capacity of the respiratory muscles (e.g. neuromuscular diseases), an increase in load (e.g. kyphoscoliosis or obstructive lung disease), or both [1]. Noninvasive mechanical ventilation (NMV), eliminates symptoms of hypoventilation and improves daytime blood gas values. Furthermore, NMV may be used outside the hospital, usually in the patient's home [2, 3]. Traditionally, volume-cycled intermittent positive pressure ventilation (IPPV) via mask has been the most frequently used mode of NMV [2, 3].

Recently, however, there has been increasing interest in noninvasive ventilation in which the airway pressure rather than the tidal volume is imposed by the ventilator: pressure-controlled ventilation (PCV). This is of proven value in the management of patients with acute respiratory failure (ARF) [4]. In the treatment of CRF, PCV has practical and economic advantages over IPPV.
In our institution, NMV was introduced in 1989. Since the introduction of PCV, anecdotal evidence has suggested that, in a number of patients, it was not possible to maintain adequate control of $\mathrm{CRF}$ with this mode of ventilation on a medium- and long-term basis. Data concerning PCV in CRF have been reported only from short-term studies (less than 1 week) involving small numbers of patients [5-7]. The comparisons between volume-controlled IPPV and PCV that have been published have also addressed only short-term use [7, 8]. Furthermore, in these studies, the ventilation modes were not standardized $[7,8]$, which raised concern that additional variables, such as assisted versus controlled ventilation, may also have influenced the results.

In order to avoid these disadvantages in the present study, we therefore compared on a medium-term basis the efficacy of two standardized NMV modes; i.e. volume-controlled IPPV and PCV. In order to eliminate 
confounding influences due to triggering, we opted to use both methods of ventilation in a controlled mode. The main difference between the two methods of ventilation is that in PCV the variable is the volume applied, whereas in IPPV the variable is the ventilator pressure. These technical properties may be responsible for a possible difference in the therapeutic efficacy of each method of ventilation. We attempted to quantify the proportion of patients in whom, despite initial satisfactory treatment with IPPV, PCV subsequently proved inadequate to maintain control of CRF. The study was open, nonrandomized and noncontrolled. This study design was chosen because our institutional review board found it unethical to withhold a treatment of proven efficacy.

\section{Materials and methods}

\section{Subjects}

The protocol was approved by our Ethics Committee, and all subjects gave written informed consent. The subjects were 30 consecutive patients ( 24 males and 6 females) with slowly progressive CRF. The demographic data, the main diagnosis, and the chronic medication used by the patients before admission are presented in table 1. Baseline values were determined for haemoglobin $(\mathrm{Hb})$, haematocrit $(\mathrm{Hct}), \mathrm{pH}$, arterial oxygen tension $\left(\mathrm{Pa}, \mathrm{O}_{2}\right)$, arterial carbon dioxide tension $\left(\mathrm{Pa}, \mathrm{CO}_{2}\right)$ at rest (breathing room air), respiratory frequency $(f \mathrm{R})$, tidal volume $(V \mathrm{~T})$, mouth occlusion pressure $\left(P_{0.1}\right)$, maximal inspiratory pressure $(P \mathrm{I}<, \max )$, vital capacity $(\mathrm{VC})$, forced expiratory volume in one second (FEV1), and the mean nocturnal oxygen saturation $\left(\mathrm{Sa}_{\mathrm{a}} \mathrm{O}_{2}\right)$ in patients at the time of admission to our hospital. All patients had been referred to us by respiratory physicians who had pretreated the patients for at least 6 months using maximum standard therapy, which also included administration of long-term oxygen (table 1).

Inclusion criteria. Only patients with slowly progressive diseases leading to chronic hypercapnic failure $\left(P \mathrm{a}, \mathrm{CO}_{2}>6.3 \mathrm{kPa}\right)$ were included. Patients suffering from obesity-hypoventilation syndrome (OHS) were included

Table 1. - Demographic data, main diagnosis, response to pressure-controlled ventilation and chronic medication of patients studied

\begin{tabular}{|c|c|c|c|c|c|c|c|}
\hline $\begin{array}{l}\mathrm{Pt} \\
\text { No. }\end{array}$ & Sex & $\begin{array}{l}\mathrm{Age} \\
\mathrm{yrs}\end{array}$ & $\begin{array}{l}\text { Height } \\
\mathrm{cm}\end{array}$ & $\begin{array}{l}\text { Weight } \\
\text { kg }\end{array}$ & $\begin{array}{c}\text { Main } \\
\text { diagnosis }\end{array}$ & $\mathrm{R} / \mathrm{NR}$ & Therapy \\
\hline 1 & M & 48 & 172 & 100 & OHS & $\mathrm{R}$ & $\mathrm{T}, \mathrm{OS}, \mathrm{D}$ \\
\hline 2 & $\mathrm{~F}$ & 20 & 138 & 27 & SMA & $\mathrm{R}$ & LTOT, T \\
\hline 3 & $\mathrm{M}$ & 54 & 173 & 53 & Post-TB & NR & LTOT, T, I $\beta$ \\
\hline 4 & $\mathrm{~F}$ & 29 & 152 & 40 & SMA & IPPV-NR & $\mathrm{T}$ \\
\hline 5 & $\mathrm{M}$ & 32 & 180 & 50 & DMD & NR & LTOT, D \\
\hline 6 & M & 75 & 169 & 72 & COPD & $\mathrm{R}$ & T, LTOT, OS, I $\beta$, IAch \\
\hline 7 & M & 38 & 176 & 94 & Scoliosis & NR & LTOT, T, D \\
\hline 8 & M & 60 & 182 & 83 & Post-TB & NR & LTOT, T, D, I $\beta$, IAch \\
\hline 9 & M & 62 & 150 & 52 & COPD & NR & T, LTOT, D, OS, I $\beta$, IAch, IS \\
\hline 10 & M & 46 & 170 & 120 & COPD & $\mathrm{R}$ & T, LTOT, D, OS, I $\beta$, IAch \\
\hline 11 & M & 63 & 175 & 64 & SMA & NR & $\mathrm{T}, \mathrm{D}$ \\
\hline 12 & $\mathrm{M}$ & 19 & 156 & 56 & DMD & NR & LTOT, T, IS \\
\hline 13 & $\mathrm{~F}$ & 61 & 167 & 47 & Post-TB & $\mathrm{R}$ & LTOT, T, IS, I $\beta$ \\
\hline 14 & M & 63 & 180 & 156 & OHS & $\mathrm{R}$ & $\mathrm{T}, \mathrm{D}, \mathrm{I} \beta, \mathrm{IS}, \mathrm{OS}$ \\
\hline 15 & M & 70 & 178 & 118 & OHS & $\mathrm{R}$ & $\mathrm{T}, \mathrm{D}, \mathrm{I} \beta, \mathrm{IAch}, \mathrm{OS}$ \\
\hline 16 & M & 66 & 178 & 79 & Post-TB & $\mathrm{R}$ & $\mathrm{T}, \mathrm{IS}, \mathrm{I} \beta$ \\
\hline 17 & $\mathrm{M}$ & 60 & 168 & 96 & OHS & $\mathrm{R}$ & LTOT, T, D, OS \\
\hline 18 & M & 72 & 160 & 65 & Scoliosis & $\mathrm{R}$ & $\mathrm{T}, \mathrm{D}, \mathrm{IS}, \mathrm{I} \beta$ \\
\hline 19 & M & 44 & 163 & 110 & PPS & $\mathrm{R}$ & LTOT, T \\
\hline 20 & M & 51 & 186 & 146 & OHS & $\mathrm{R}$ & $\mathrm{T}, \mathrm{D}, \mathrm{I} \beta, \mathrm{OS}$ \\
\hline 21 & M & 33 & 178 & 150 & OHS & $\mathrm{R}$ & $\mathrm{T}, \mathrm{D}, \mathrm{I} \beta$, IAch, OS \\
\hline 22 & $\mathrm{~F}$ & 37 & 140 & 57 & PPS & $\mathrm{R}$ & $\mathrm{T}, \mathrm{I} \beta, \mathrm{OS}$ \\
\hline 23 & $\mathrm{~F}$ & 33 & 155 & 47 & Scoliosis & $\mathrm{R}$ & $\mathrm{T}, \mathrm{D}, \mathrm{IS}, \mathrm{OS}$ \\
\hline 24 & $\mathrm{~F}$ & 72 & 164 & 63 & Post-TB & $\mathrm{R}$ & LTOT, T, D \\
\hline 25 & M & 64 & 170 & 85 & Post-TB & NR & $\mathrm{D}, \mathrm{OS}$ \\
\hline 26 & $\mathrm{M}$ & 60 & 170 & 106 & OHS & $\mathrm{R}$ & $\mathrm{T}, \mathrm{D}$ \\
\hline 27 & $\mathrm{M}$ & 52 & 178 & 74 & Scoliosis & NR & LTOT, T, D \\
\hline 28 & M & 44 & 169 & 115 & OHS & IPPV-NR & $\mathrm{T}, \mathrm{D}, \mathrm{I} \beta$, IAch, OS \\
\hline 29 & $\mathrm{M}$ & 68 & 148 & 48 & Post-TB & $\mathrm{R}$ & LTOT, T, D, I $\beta$, IAch \\
\hline 30 & M & 70 & 167 & 69 & Post-TB & NR & $\mathrm{T}, \mathrm{D}, \mathrm{OS}$ \\
\hline Mean & & 52 & 167 & 81 & & & \\
\hline $\mathrm{SD}$ & & 16 & 12 & 34 & & & \\
\hline
\end{tabular}

Pt: patient; M: male; F: female; R: responder; NR: nonresponder; IPPV: intermittent positive pressure ventilation; OHS: obesityhypoventilation syndrome; COPD: chronic obstructive pulmonary disease; DMD: Duchenne's muscle dystrophy; PPS: postpolio syndrome; Post-TB: post-tuberculosis sequelae; SMA: spinal muscular atrophy; T: theophylline; OS: oral steroids; D: diuretics; LTOT: long-term oxygen therapy; $\mathrm{I} \beta$ : inhaled betamimetic agents; IAch: inhaled anticholinergic agents; IS: inhaled steroids. 
in the study if correction of the sleep-related breathing disturbance was not obtained after 1 week of nasal continuous positive airway pressure (CPAP) therapy.

Exclusion criteria. Exclusion criteria included acute respiratory failure during the 4 weeks prior to admission to the hospital, acute bronchopulmonary infection, fever, haemodynamic instability, severe acidosis, malignant arterial hypertension, lack of co-operation, or rapidly progressive neuromuscular diseases (e.g. progressive amyotrophic lateral sclerosis).

\section{Materials}

Body plethysmography was performed using the Masterlab (E. Jäger, Würzburg, Germany) normal values [9]. $V \mathrm{~T}$ and $f \mathrm{R}$ were measured with a portable pneumotachograph (CP100; Bicore, Medilab, Estenfeld, Germany). Samples for capillary blood gas analysis (Gas Check; AVL, Bad Homburg, Germany) were obtained from the hyperaemic earlobe. Oxygen saturation was measured with a pulse oximeter (Pulsox 7; Minolta, AVL, Bad Homburg, Germany). A separate unit (hardware and software) for continuous registration of $P 0.1$ and $P$ I,max was manufactured by one of our engineers (M. Klauke). $P_{0.1}$ was measured as described by WhITELAW et al. [10]. PI,max was determined according to the method of BLACK and Hyatт [11].

\section{Symptom scores}

Dyspnoea during rest was measured based on the Borg scale [12]. This scale ranges from 0 (not short of breath) to 10 (extremely short of breath). The symptom scores for morning headache, sleep quality, and mobility, and the nuisance scores associated with use of the ventilator (i.e. noise, alarms, or weight of the device) were recorded on the basis of a standardized visual analogue scale (0-10; in which the larger numerical values represented worsening symptoms).

\section{Study protocol}

The study was conducted on a prospective basis. Preexisting treatment, including pharmacotherapy and longterm oxygen therapy (LTOT) were continued during the daytime when the patients did not use the ventilator. Oxygen insufflation was discontinued $1 \mathrm{~h}$ before all measurements were taken. All objective variables and symptom scores were measured at baseline, and 1, 2 and 6 months after NMV. The blood gas values were measured on two consecutive days, six times altogether (each day at 09:00, 14:00 and 18:00 h). The mean value was taken. The other measurements were performed during spontaneous breathing $8-10 \mathrm{~h}$ after discontinuation of nocturnal mechanical ventilation. The ventilator settings were adjusted when patients were awake. During the daytime adaptation the patients had to fulfil three conditions in order to be judged as acclimatizing adequately to NMV: 1) acceptance of NMV in the controlled mode without spontaneous breathing activity (which was assessed visually by an experienced therapist); 2) normocapnia during daytime NMV; and 3) no relevant air leakage from the mask. During the adaptation phase, the patients were sitting or in a semirecumbent posture. Blood gas values were measured after 15 and $30 \mathrm{~min}$ of NMV.

For each patient, the study was considered to have started when the adjustment to IPPV had been completed. The quality of nocturnal IPPV was evaluated by recording the $\mathrm{S}_{\mathrm{a}} \mathrm{O}_{2}$. The patients were readmitted to hospital after 1 month of nocturnal IPPV. The symptom scores and the objective parameters described above were re-evaluated at that point. If the measurements taken after this IPPV period showed an improvement and the patient agreed to continue the study, the patient then converted to PCV without any treatment-free interval. The quality of nocturnal PCV was evaluated again and documented by recording the $\mathrm{Sa}, \mathrm{O}_{2}$ with a pulse oximeter.

At the end of the 4 week PCV period, two groups were defined ("responders" and "nonresponders") on the basis of their symptom scores and $\mathrm{Pa}, \mathrm{O}_{2}$ values. Responders to PCV showed no worsening in their symptom score and were defined as those with maintenance of normocapnia. These patients continued with PCV for the following 4 months. Nonresponders to PCV were defined as those with a deterioration in their symptom scores (of more than 1 point), and/or an increase in $\mathrm{Pa}, \mathrm{O}_{2}(>0.6$ $\mathrm{kPa}$ ) compared to the IPPV period. Nonresponders returned to IPPV. A final assessment was made 4 months later.

\section{Ventilators}

For IPPV, the Dräger EV 800 (Dräger, Lübeck, Germany; weight $16.5 \mathrm{~kg}$ ) and the PLV 100 (Lifecare, Denver, CO, USA; weight $12.8 \mathrm{~kg}$ ) were used. The price of these devices was DM 20,000-22,000 (US\$ 13,500-15,000). The trigger threshold was reduced, so that the respirators were always used in the controlled mode. For PCV, the bilevel positive airway pressure (BiPAP) was used in T-mode (Respironics Inc., Murrysville, PA, USA; weight $4.3 \mathrm{~kg}$ ). The cost of this device was approximately DM 16,500 (US\$ 11,500).

\section{Ventilator settings}

Before IPPV was started, $f \mathrm{R}$ of the spontaneously breathing patient was determined using a portable pneumotachograph. The ventilator rate was set at the same rate or slightly higher than that measured during spontaneous breathing. The duty cycle ratio (fraction of inspiration to total duration of respiratory cycle $(t \mathrm{I} / t$ tot $))$ was chosen according to the underlying disease and the patients' subjective well-being (in restrictive thoracic diseases, the inspiration time was typically set between 0.4 and $0.5 \mathrm{~s}$, in OHS patients, between 0.3 and $0.5 \mathrm{~s}$, and in chronic obstructive pulmonary disease (COPD) patients between 0.3 and $0.45 \mathrm{~s}$ ). The PCV ventilator rate was set at the same or a slightly higher rate than that measured during spontaneous breathing. The inspiratory time was chosen as mentioned above. The $V$ T determined 
during the preceding IPPV phase was taken as the reference value for the ensuing PCV phase. In order to monitor $V \mathrm{~T}$, a pneumotachograph was inserted in the tubing system. The inspiratory positive airway pressure (IPAP) was adjusted so that the known $V$ T was achieved. Expiratory positive airway pressure (EPAP) in patients with restrictive thoracic disease and OHS was set as low as possible (at $2 \mathrm{cmH}_{2} \mathrm{O}$ ). In patients with COPD, EPAP was chosen between 2.5 and $4 \mathrm{cmH}_{2} \mathrm{O}$.

\section{Statistical analysis}

Results are expressed as mean \pm SD. The significance for differences between matched pairs was determined by the Wilcoxon test (two-sided). The significance for intraindividual multiple comparisons was confirmed using Newman-Keul analysis [13]. Differences between the responder and nonresponder group were determined by the Mann-Whitney test (two-sided). In all cases, a p-value of less than 0.05 was considered to be significant.

\section{Results}

All patients had CRF, characterized by hypercapnia, hypoxaemia and persistent nocturnal oxygen desaturation (table 2). At baseline, $P_{0.1}, P_{0.1} / P_{\mathrm{I}}$,max, and $f \mathrm{R}$ were increased in all patients, whereas $\mathrm{pH}, P \mathrm{I}$,max, $V \mathrm{~T}, \mathrm{VC}$, FEV1 and $\mathrm{Sa}_{\mathrm{a}} \mathrm{O}_{2}$ were reduced in relation to the predicted

Table 2. - Measurements at baseline and after 1 month of IPPV in all patients

\begin{tabular}{|c|c|c|c|}
\hline & Baseline & p-value & $\begin{array}{c}\text { IPPV } \\
\text { after } 1 \text { month }\end{array}$ \\
\hline $\mathrm{Hb} \mathrm{mmol} \cdot \mathrm{L}^{-1}$ & $10.2 \pm 1.6$ & NS & $9.9 \pm 1.2$ \\
\hline Het \% & $50 \pm 8$ & * & $48 \pm 5$ \\
\hline $\mathrm{pH}$ & $7.33 \pm 0.04$ & $* * *$ & $7.38 \pm 0.03$ \\
\hline $\mathrm{Pa}_{\mathrm{a}} \mathrm{O}_{2} \mathrm{kPa}$ & $6.4 \pm 1.2$ & $* * *$ & $8.3 \pm 1.0$ \\
\hline$P \mathrm{a}, \mathrm{CO}_{2} \mathrm{kPa}$ & $7.5 \pm 0.9$ & $* * *$ & $5.5 \pm 0.8$ \\
\hline$P_{0.1} \mathrm{cmH}_{2} \mathrm{O}$ & $4.1 \pm 1.4$ & $* * *$ & $2.9 \pm 0.9$ \\
\hline$P \mathrm{I}, \max \mathrm{cmH}_{2} \mathrm{O}$ & $40.5 \pm 12.8$ & $* * *$ & $56.5 \pm 13.6$ \\
\hline$P_{0.1} / P_{\mathrm{I}, \max }$ & $11.7 \pm 4.6$ & $* * *$ & $5.3 \pm 2.1$ \\
\hline$f \mathrm{R}$ breaths $\cdot \mathrm{min}^{-1}$ & $25.6 \pm 5.2$ & $* * *$ & $19.2 \pm 3.3$ \\
\hline$V \mathrm{~T} \mathrm{~mL}$ & $341 \pm 110$ & ** & $440 \pm 152$ \\
\hline VC L & $1.6 \pm 0.6$ & NS & $1.6 \pm 0.6$ \\
\hline FEV1 L & $1.01 \pm 0.4$ & NS & $1.1 \pm 0.4$ \\
\hline $\mathrm{Sa}_{\mathrm{a}, \mathrm{O}_{2}} \%$ & $77 \pm 6$ & $* * *$ & $93 \pm 2$ \\
\hline Dyspnoea & $5.6 \pm 2.6$ & $* * *$ & $1.3 \pm 0.9$ \\
\hline Headache & $3.2 \pm 3.2$ & $* * *$ & $0.4 \pm 0.8$ \\
\hline Sleep quality & $6.8 \pm 2.3$ & $* * *$ & $1.6 \pm 0.8$ \\
\hline Mobility & $7.5 \pm 1$ & $* * *$ & $2.5 \pm 0.8$ \\
\hline Noise (device) & - & - & $2.3 \pm 2.4$ \\
\hline Weight (device) & - & - & $5.1 \pm 3.2$ \\
\hline Alarm (device) & - & - & $3.5 \pm 2.4$ \\
\hline
\end{tabular}

Values are presented as mean \pm SD. IPPV: intermittent positive pressure ventilation; $P \mathrm{a}, \mathrm{O}_{2}$ : arterial oxygen tension; $P_{\mathrm{a}, \mathrm{CO}_{2}}$ : arterial carbon dioxide tension; Hb: haemoglobin; Hct: haematocrit; $P_{0.1}$ : occlusion pressure; $P_{\mathrm{I}, \mathrm{max}}$ : maximal inspiratory pressure; $f \mathrm{R}$ : respiratory frequency; $V \mathrm{~T}$ : tidal volume; $\mathrm{VC}$ : vital capacity; FEV1: forced expiratory volume in one second; $\mathrm{Sa}_{\mathrm{a}} \mathrm{O}_{2}$ : arterial oxygen saturation. $*$ : $\mathrm{p}<0.05 ; * *$ : $\mathrm{p}<0.01 ;{ }^{* * *}$ : $\mathrm{p}<0.001$; NS: nonsignificant, comparing measurement at baseline and after 1 month of IPPV.

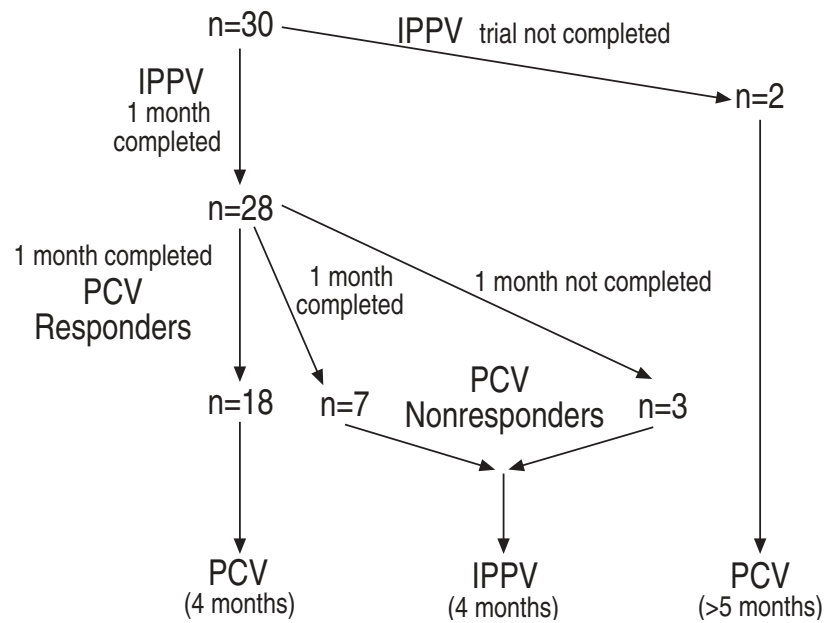

Fig. 1. - Flow chart of the study involving a total of 30 patients. IPPV: intermittent positive pressure ventilation; PCV: pressure-controlled ventilation.

value (table 2). All patients showed general disability indicated by the symptom scores (table 2).

During the IPPV phase, two patients (Nos. 19 and 20) did not tolerate IPPV due to adverse gastrointestinal effects, such as flatulence. These adverse effects led to premature discontinuation of IPPV after 14 and 16 days. Both patients were viewed as nonresponders to IPPV (fig. 1). Treatment with PCV was successful in both patients, with daytime normalization of hypercapnia achieved during spontaneous breathing within the next 2 weeks.

After 1 month of IPPV, the following values changed significantly in all patients: $P_{\mathrm{a}}, \mathrm{CO}_{2}$, Hct, $f \mathrm{R}, P_{0.1}$, and $P 0.1 / P \mathrm{i}$,max decreased; $\mathrm{Pa}_{\mathrm{a}} \mathrm{O}_{2}, P \mathrm{I}$,max, $V \mathrm{~T}$, and $\mathrm{pH}$ increased, while $\mathrm{Hb}, \mathrm{VC}$ and FEV1 remained unchanged (table 2). Simultaneously, the symptom scores improved (table 2).

The disturbance to all patients caused by the weight and the alarms of the IPPV ventilators was significantly greater when compared with the PCV phase. The visual analogue scores for the weight of the ventilator were $5.1 \pm 3.2$ for IPPV and $1.0 \pm 1.5$ for PCV. The mean score for the alarm was $3.5 \pm 2.4$ for IPPV, while there were no alarms associated with the use of PCV. With regard to the noise of the ventilators, no significant difference could be found between the units $(2.3 \pm 2.4$ versus 2.3 \pm 2.6 ).

During the subsequent PCV phase, three patients discontinued either after the first study night (patient No. 21) or after four and six nights of treatment (patients Nos. 24 and 30), because they felt subjectively worse. They complained about morning dyspnoea, and deterioration of mobility and sleep quality. All three patients successfully continued with IPPV, without further adverse effects. The remaining 25 patients completed the 1 month period of PCV (fig. 1). The mean $\mathrm{Sa}_{2} \mathrm{O}_{2}$ during nocturnal PCV was $92 \pm 2 \%$. At the end of the PCV period the patients were classified as responders or nonresponders depending on the subjective scoring and the $\mathrm{Pa}_{\mathrm{a}} \mathrm{CO}_{2}$ values (see Methods).

Eighteen patients responded to PCV (fig. 1). Compared with the preceding IPPV phase, the objective parameters in the responders improved further or remained the same: Hct, $P_{\mathrm{a}}, \mathrm{CO}_{2}, P_{0.1}$ and $P_{0.1} / P_{\mathrm{I}}$,max decreased; $P \mathrm{I}$,max and $V \mathrm{~T}$ increased; $\mathrm{Hb}, \mathrm{pH}, \mathrm{Pa}_{\mathrm{a}}, \mathrm{O}_{2}, \mathrm{R}, \mathrm{VC}$ and $\mathrm{FEV} 1$ did not change significantly (tables 3 and 4 ). The symptom scores 
Table 3. - Haemoglobin, haematocrit, blood gas values and arterial oxygen saturation measured at baseline, after 1 month of IPPV, after a second month of PCV, and after 6 months of NMV

\begin{tabular}{|c|c|c|c|c|c|c|c|}
\hline & \multirow[b]{2}{*}{ Baseline } & \multicolumn{2}{|c|}{ After IPPV } & \multicolumn{2}{|c|}{ After PCV } & \multicolumn{2}{|c|}{ After NMV } \\
\hline & & p-value & At 1 month & p-value & At 2 months & p-value & At 6 months \\
\hline \multicolumn{8}{|l|}{ Responders } \\
\hline Patient $\mathrm{n}$ & 18 & & 18 & & 18 & & 18 \\
\hline $\mathrm{Hb} \mathrm{mmol} \cdot \mathrm{L}^{-1}$ & $16.3 \pm 3.0$ & NS & $9.9 \pm 1.4$ & NS & $9.5 \pm 0.8$ & NS & $9.5 \pm 1.0$ \\
\hline Hct \% & $50 \pm 9$ & $*$ & $49 \pm 7$ & $*$ & $46 \pm 4$ & NS & $45 \pm 5$ \\
\hline $\mathrm{pH}$ & $7.34 \pm 0.09$ & $* *$ & $7.38 \pm 0.03$ & NS & $7.39 \pm 0.04$ & NS & $7.39 \pm 0.02$ \\
\hline $\mathrm{Pa}_{\mathrm{a}, \mathrm{O}_{2}} \mathrm{kPa}$ & $6.6 \pm 0.8$ & $* * *$ & $8.0 \pm 0.9$ & NS & $8.3 \pm 1.0$ & NS & $8.1 \pm 1.2$ \\
\hline 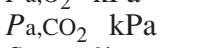 & $7.1 \pm 0.6^{\#}$ & $* * *$ & $5.5 \pm 0.5$ & $*$ & $5.4 \pm 0.5^{\# \# \# ~}$ & NS & $5.6 \pm 0.5$ \\
\hline $\mathrm{Sa}_{\mathrm{a}, \mathrm{O}_{2}} \%$ & $80 \pm 5^{\#}$ & $* * *$ & $93 \pm 2$ & NS & $93 \pm 2.3$ & NS & $93 \pm 2$ \\
\hline \multicolumn{8}{|l|}{ Nonresponders } \\
\hline Patient $\mathrm{n}$ & 10 & & 10 & & 7 & & 10 \\
\hline $\mathrm{Hb} \mathrm{mmol} \cdot \mathrm{L}^{-1}$ & $10.7 \pm 0.9$ & $*$ & $10.2 \pm 0.7$ & NS & $9.7 \pm 0.7$ & NS & $9.8 \pm 0.5$ \\
\hline Hct \% & $52 \pm 4.4$ & * & $49 \pm 3$ & NS & $47 \pm 4$ & NS & $46 \pm 3$ \\
\hline $\mathrm{pH}$ & $7.32 \pm 0.03$ & $* *$ & $7.37 \pm 0.03$ & NS & $7.36 \pm 0.01$ & NS & $7.39 \pm 0.02$ \\
\hline $\mathrm{Pa}_{\mathrm{a}, \mathrm{O}_{2}} \mathrm{kPa}$ & $5.7 \pm 1.4$ & $* *$ & $8.5 \pm 0.5$ & * & $8.0 \pm 0.6$ & NS & $8.4 \pm 1.3$ \\
\hline$P \mathrm{a}, \mathrm{CO}_{2} \quad \mathrm{kPa}$ & $8.1 \pm 1$ & $* *$ & $5.7 \pm 0.4$ & * & $6.6 \pm 0.5$ & $*$ & $5.5 \pm 0.5$ \\
\hline $\mathrm{S}_{\mathrm{a}, \mathrm{O}_{2}}{ }^{2} \%$ & $7.5 \pm 6$ & $* *$ & $93 \pm 2$ & NS & $92 \pm 2$ & NS & $92 \pm 2$ \\
\hline
\end{tabular}

Values are presented as mean \pm SD. PCV: pressure-cycled ventilation; NMV: noninvasive mechanical ventilation. For further definitions see legends to tables 1 and 2. Significant differences within the responder and nonresponder group: *: $p<0.05 ; * *: p<0.01$; ***: $\mathrm{p}<0.001$. Significant differences between responder and nonresponder: \#: $\mathrm{p}<0.05$; \#\#: $\mathrm{p}<0.01$; \#\#\#: $\mathrm{p}<0.001$.

Table 4. - $P_{0.1}, P I, \max , f^{\prime} R, V T, V C$ and FEV 1 measured at baseline, after 1 month of IPPV, after a second month of PCV, and after 6 months of NMV

\begin{tabular}{|c|c|c|c|c|c|c|c|}
\hline & \multirow[b]{2}{*}{ Baseline } & \multicolumn{2}{|c|}{ After IPPV } & \multicolumn{2}{|c|}{ After PCV } & \multicolumn{2}{|c|}{ After NMV } \\
\hline & & p-value & At 1 month & p-value & At 2 months & p-value & At 6 months \\
\hline \multicolumn{8}{|l|}{ Responders } \\
\hline Patient $n$ & 18 & & 18 & & 18 & & 18 \\
\hline$P 0.1 \quad \mathrm{cmH}_{2} \mathrm{O}$ & $4.4 \pm 1.6$ & $* *$ & $3.2 \pm 1$ & * & $2.9 \pm 1$ & NS & $2.9 \pm 0.8$ \\
\hline$P_{\mathrm{I}, \max } \mathrm{cmH}_{2} \mathrm{O}$ & $40.1 \pm 13.2$ & $* * *$ & $56.2 \pm 12.7$ & $*$ & $58.1 \pm 20.3$ & NS & $60.1 \pm 17.9$ \\
\hline$P_{0.1 / P_{\mathrm{I}, \max }}$ & $12.2 \pm 6.2$ & $* * *$ & $6.0 \pm 2.2$ & * & $5.2 \pm 2.3$ & NS & $5.1 \pm 2.1$ \\
\hline$f^{\prime} \mathrm{R}$ breaths $\cdot \min ^{-1}$ & $25.5 \pm 5.2$ & $* * *$ & $19 \pm 3.3$ & NS & $18.7 \pm 3.4$ & NS & $18.3 \pm 2.9$ \\
\hline$V \mathrm{~T} \quad \mathrm{~mL}$ & $350 \pm 117$ & $* *$ & $467 \pm 188$ & * & $531 \pm 193$ & NS & $550 \pm 186$ \\
\hline VC L & $1.8 \pm 0.8$ & NS & $1.8 \pm 0.8$ & NS & $1.9 \pm 0.8$ & NS & $1.8 \pm 0.8$ \\
\hline FEV1 L & $1.1 \pm 0.5$ & $*$ & $1.3 \pm 0.5$ & NS & $1.3 \pm 0.6$ & NS & $1.2 \pm 0.5$ \\
\hline \multicolumn{8}{|l|}{ Nonresponders } \\
\hline Patient $\mathrm{n}$ & 10 & & 10 & & 7 & & 10 \\
\hline$P_{0.1} \mathrm{cmH}_{2} \mathrm{O}$ & $4.1 \pm 1.6$ & $* *$ & $2.7 \pm 1$ & NS & $3.5 \pm 1.1$ & NS & $2.6 \pm 0.8$ \\
\hline$P \mathrm{I}, \max \mathrm{cmH}_{2} \mathrm{O}$ & $39.5 \pm 14.6$ & $* *$ & $60.9 \pm 15.6$ & NS & $64.4 \pm 15.9$ & NS & $63.4 \pm 18.3$ \\
\hline$P 0.1 / P \mathrm{I}, \max$ & $11.1 \pm 2.9$ & $* *$ & $4.3 \pm 1$ & NS & $5.5 \pm 1.5$ & NS & $3.9 \pm 0.7$ \\
\hline$f^{\prime} \mathrm{R}$ breaths $\cdot \min ^{-1}$ & $26 \pm 5.9$ & $* *$ & $18.7 \pm 3$ & NS & $22 \pm 3.6$ & NS & $19.7 \pm 3.3$ \\
\hline$V \mathrm{~T} \quad \mathrm{~mL}$ & $340 \pm 97$ & * & $447 \pm 100$ & NS & $404 \pm 78$ & NS & $467 \pm 93$ \\
\hline VC L & $1.5 \pm 0.3$ & NS & $1.6 \pm 0.3$ & NS & $1.6 \pm 0.3$ & NS & $1.6 \pm 0.4$ \\
\hline FEV1 L & $1.0 \pm 0.3$ & NS & $1.1 \pm 0.4$ & NS & $1.0 \pm 0.3$ & NS & $1.1 \pm 0.4$ \\
\hline
\end{tabular}

Values are presented as mean \pm sD. For definitions see legends to tables 2 and 3. Significant difference within the responder and nonresponder group: $*: \mathrm{p}<0.05 ; * *: \mathrm{p}<0.01 ; * * *: \mathrm{p}<0.001$.

with regard to dyspnoea, headache, mobility and sleep remained unchanged (table 5). At the end of the PCV phase, the responders continued with PCV. The important advantages from the patients' point of view were the absence of alarms and the lesser weight of the PCV unit (table 5). Table 6 shows the ventilator settings in responders and nonresponders.

After 1 month of PCV, the symptom scores of the 10 nonresponders, with regard to dyspnoea, sleep quality and mobility, worsened significantly compared with the preceding IPPV phase and with the responder group (table 5). Nevertheless, at the end of the PCV period the average scores were still significantly better than baseline values (table 5). Furthermore, all nonresponders could be clearly identified by a significant increase in $\mathrm{Pa}, \mathrm{CO}_{2}$ (fig. 2) and a corresponding decrease in $\mathrm{Pa}, \mathrm{O}_{2}$ (table 4 ). The other objective variables did not change significantly (tables 3 and 4). The likelihood of being a nonresponder was not related to the underlying diagnosis. At baseline, compared to responders, nonresponders had lower mean nocturnal $\mathrm{Sa}_{\mathrm{a}} \mathrm{O}_{2}(\mathrm{p}<0.05)$ and a higher $\mathrm{Pa}_{\mathrm{a}} \mathrm{CO}_{2}(\mathrm{p}<0.05)($ table 3$)$.

After completion of the PCV phase, all nonresponders returned to the initial IPPV therapy. During the ensuing 4 months on IPPV, nonresponders regained daytime normocapnia (fig. 2) and showed significant improvements in dyspnoea, sleep quality, and mobility (tables 3 and 5). However, the weight and the alarms of the IPPV ventilators again caused high symptom scores in the nonresponder group (table 5). 
Table 5. - Symptom scores measured at baseline, after 1 month of IPPV, after a second month of PCV, and after 6 months of NMV

\begin{tabular}{|c|c|c|c|c|c|c|c|}
\hline & \multirow[b]{2}{*}{ Baseline } & \multicolumn{2}{|c|}{ After IPPV } & \multicolumn{2}{|c|}{ After PCV } & \multicolumn{2}{|c|}{ After NMV } \\
\hline & & p-value & At 1 month & p-value & At 2 months & $\mathrm{p}$-value & At 6 months \\
\hline \multicolumn{8}{|l|}{ Responders } \\
\hline Patient $\mathrm{n}$ & 18 & & 18 & & 18 & & 18 \\
\hline Dyspnoea & $5.4 \pm 3.0$ & $* * *$ & $1.1 \pm 1.2$ & NS & $1.1 \pm 1.2^{\# \#}$ & NS & $0.7 \pm 0.9$ \\
\hline Headache & $2.9 \pm 3.2$ & $* * *$ & $0.0 \pm 0.0$ & NS & $0.0 \pm 0.0$ & NS & $0.0 \pm 0.0$ \\
\hline Sleep quality & $6.5 \pm 2.2$ & $* * *$ & $1.5 \pm 0.8$ & NS & $1.1 \pm 1^{\# \#}$ & NS & $0.7 \pm 0.9$ \\
\hline Mobility & $7.3 \pm 1.1$ & $* * *$ & $2.6 \pm 1.0$ & NS & $2.1 \pm 1.1^{\# \#}$ & NS & $1.7 \pm 0.7$ \\
\hline Noise (device) & - & - & $2.4 \pm 1.6$ & NS & $2.3 \pm 2.4$ & NS & $1.9 \pm 2.0$ \\
\hline Weight (device) & - & - & $5.6 \pm 2.8$ & $* * *$ & $1.2 \pm 2.0$ & NS & $1.2 \pm 1.1$ \\
\hline Alarm (device) & - & - & $3.8 \pm 2.4$ & - & - & - & - \\
\hline \multicolumn{8}{|l|}{ Nonresponders } \\
\hline Patient $\mathrm{n}$ & 10 & & 10 & & 7 & & 10 \\
\hline Dyspnoea & $5.8 \pm 2.4$ & $* *$ & $1.4 \pm 0.7$ & * & $3.1 \pm 1.0$ & * & $1.3 \pm 0.8$ \\
\hline Headache & $3.3 \pm 2.9$ & $* *$ & $0.9 \pm 1.0$ & NS & $0.1 \pm 0.3$ & NS & $0.2 \pm 0.4$ \\
\hline Sleep quality & $6.8 \pm 2.7$ & $* *$ & $1.7 \pm 0.8$ & $*$ & $4.0 \pm 1.3$ & NS & $0.9 \pm 0.7$ \\
\hline Mobility & $7.7 \pm 0.8$ & ** & $2.4 \pm 0.7$ & * & $4.8 \pm 1.2$ & * & $2.1 \pm 0.6$ \\
\hline Noise (device) & - & & $2.2 \pm 3.3$ & NS & $2.3 \pm 2.9$ & NS & $0.5 \pm 0.8$ \\
\hline Weight (device) & - & & $4.2 \pm 3.4$ & $*$ & $0.7 \pm 1.2$ & * & $4.0 \pm 3.4$ \\
\hline Alarm (device) & - & & $3.6 \pm 2.6$ & - & - & - & $3.4 \pm 2.5$ \\
\hline
\end{tabular}

Values are presented as mean \pm SD. For definitions see legends to tables 2 and 3. Significant differences within the responder and nonresponder group: *: $\mathrm{p}<0.05 ; * *: \mathrm{p}<0.01 ; * * *: \mathrm{p}<0.001$. Significant differences between responder and nonresponder: \#: $\mathrm{p}<0.05$; \#\#: p<0.01; \#\#: p<0.001.

Table 6. - Ventilator setting with intermittent positive pressure ventilation (IPPV) and pressure-cycled ventilation (PVC)

\begin{tabular}{lcccc}
\hline PCV settings & $\begin{array}{c}\mathrm{IPAP} \\
\mathrm{cmH}_{2} \mathrm{O}\end{array}$ & $\begin{array}{c}\mathrm{EPAP} \\
\mathrm{cmH}_{2} \mathrm{O}\end{array}$ & $\begin{array}{c}\mathrm{VR} \\
1 \mathrm{~min}^{-1}\end{array}$ & $\begin{array}{c}t \mathrm{I} \\
\%\end{array}$ \\
\hline Responders & $18.7 \pm 3.4$ & $3.3 \pm 1.6$ & $19.3 \pm 2.3$ & $42 \pm 4$ \\
Nonresponders & $17.3 \pm 5.5$ & $2.4 \pm 1.1$ & $20.7 \pm 3.7$ & $42 \pm 6$ \\
\hline IPPV settings & $\mathrm{VVT}$ & \multicolumn{2}{|l}{} & I-time \\
& $\mathrm{mL}$ & $1 \mathrm{~min}^{-1}$ & $\mathrm{~s}$ \\
\hline Responders & $690 \pm 155$ & $20.4 \pm 3$ & $1.1 \pm 0.2$ \\
Nonresponders & $709 \pm 143$ & $21 \pm 4.6$ & $1.2 \pm 0.3$ \\
\hline
\end{tabular}

EPAP: expiratory positive airway pressure; IPAP: inspiratory positive airway pressure; I-time: inspiration time; $t \mathrm{I}$ : inspiration time as a percentage of the total beathing cycle; VVT: tidal volume of the ventilator; VR: ventilator rate.

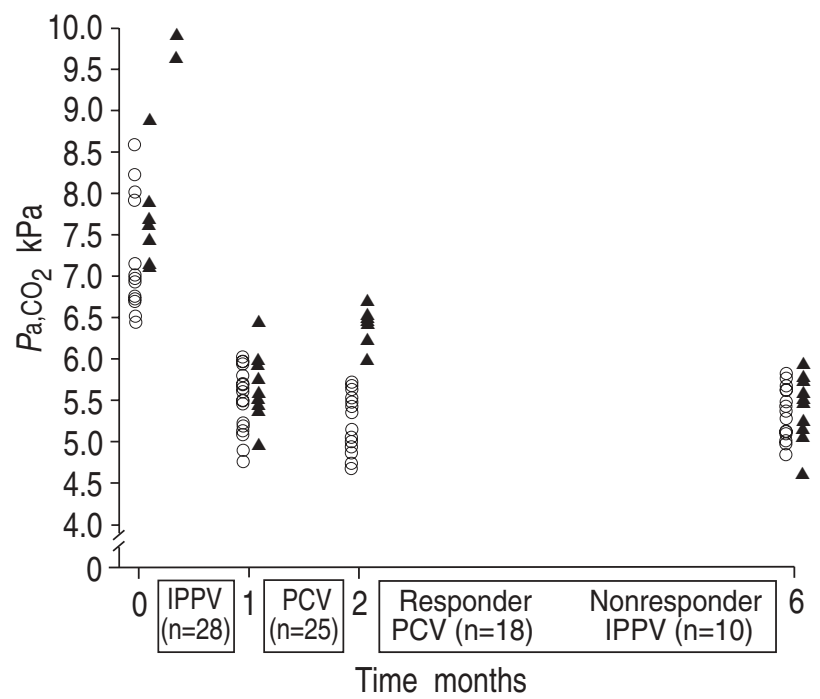

Fig. 2. - $P \mathrm{a}_{1} \mathrm{CO}_{2}$ of the responders (circles) and nonresponders (triangles) to pressure-controlled ventilation (PCV) during the course of the study. Individual $\mathrm{Pa}_{\mathrm{a}} \mathrm{CO}_{2}$ values of single patients are given as a mean of three measurements during daytime spontaneous breathing. The PCV trial, which was completed after 2 months, distinguished responders from nonresponders.

\section{Discussion}

The main finding of this study is that PCV can maintain stability in the majority of a heterogeneous population of patients with CRF after initial treatment with IPPV. In the PCV nonresponders, IPPV was able to improve the CRF-associated symptoms and hypercapnia. No patient found to be a responder after 1 month of PCV subsequently became a nonresponder.

As one would expect, with the exception of two, all patients in our study responded positively to a 1 month phase of nocturnal IPPV. These two were nonresponders to IPPV because of intractable gastrointestinal flatulence; they were adequately treated with PCV. With IPPV, all other patients showed a marked improvement in their symptom scores and in the objective respiratory parameters, a fact which was confirmed by daytime normalization of the $\mathrm{Pa}, \mathrm{CO}_{2}$ values.

After an overall improvement with IPPV in the first month of the study, two thirds of the remaining 28 patients responded positively to PCV. The nonresponders deteriorated with regard to symptoms and $\mathrm{Pa}, \mathrm{CO}_{2}$ reflecting inadequate NMV. All nonresponders returned to the initial IPPV mode after the PCV phase. Thereafter, they regained normocapnia, and the symptom scores improved in the ensuing 4 months of IPPV therapy.

Compared to the responders, nonresponders to PCV had a lower mean nocturnal $\mathrm{Sa}_{\mathrm{a}} \mathrm{O}_{2}$ and a higher mean $P \mathrm{a}, \mathrm{CO}_{2}$ at baseline, indicating more severe CRF. The other parameters did not differ significantly prior to the PCV trial. However, the above-mentioned differences in $\mathrm{Sa}, \mathrm{O}_{2}$ and $\mathrm{Pa}_{\mathrm{a}} \mathrm{CO}_{2}$ were only moderate, and there were no clear cut-off predictive values which were able to distinguish responders from nonresponders. Nevertheless, this observation is of importance and suggests that PCV should be introduced in a carefully supervised manner in patients with severe CRF.

Recently, noninvasive pressure support ventilation has been used more frequently both in patients with ARF 
[4, 14] and in CRF [5-8]. In an open study (data unpublished), we had previously found that when used on a long-term basis a number of patients who initially had a good response to PCV in the assisted mode subsequently developed a recurrence of hypercapnia and symptoms of CRF. For these patients, we were obliged to return to a controlled ventilation mode (volume- or pressure-cycled). In most cases, satisfactory treatment of CRF was then obtained. To the best of our knowledge, there are no studies in the literature which address the relative efficacy of PCV and IPPV in the medium term. Previous studies have compared IPPV and PCV only in the short term; none of these have extended for more than 1 week [5-8]. In the present study, two thirds of the nonresponders appeared after the first week. Moreover, it should be recalled that only one third of the total population were nonresponders. From the present data, it is likely that the latter studies may have missed a significant number of medium term nonresponders. Overlooking them is of clinical importance, since if not carefully monitored they could develop overt ventilatory failure after discharge from the hospital.

A randomized, cross-over design would have been preferred. Since at presentation all patients suffered from CRF our institutional review board did not permit this, and also considered a run-in period unethical because it would have required withholding an effective treatment from symptomatic patients, which may have exposed them to the risk of further deterioration. For similar reasons, we were not permitted to include a wash-out period after the 4 week period of IPPV. Since baseline measurements for the PCV without any treatment-free interval do not exist, we cannot theoretically exclude a prolonged effect of IPPV on the initial phase of the PCV. On the other hand, in all nonresponders, the deterioration of the symptom scores and the recurrence of hypercapnia occurred during the 4 week period, suggesting that any carry-over effect was transient. Moreover, it has been shown that after withdrawal from NMV, deterioration occurred after 1 week [5]. Finally, in order to exclude time effects, the design of the present study incorporated a follow-up of the patients over a 6 month period. We acknowledge that the design of this study, which was open, nonrandomized and noncontrolled, is inadequate to quantify the true prevalence of the medium- or long-term nonresponders. Nevertheless, the data do demonstrate that the problem of mediumterm nonresponders exists; and this has not previously been documented. This study could, therefore, be viewed as a pilot study, as the present data did show that the majority of patients were responders to PCV and additionally that two patients were nonresponders to IPPV. Therefore, we are convinced that a prospective randomized study of the medium- and long-term efficacy of PCV in comparison to IPPV is now warranted and would not now be unethical.

When considering the present study in comparison with previously published work, we obtain both confirmatory and cautionary conclusions. Thus, we agree that pressure preset ventilation may be an alternative to volume preset ventilation $[7,8]$. The data confirm that the majority of patients with CRF may be adequately treated with PCV. However, they also suggest that a period of 1 week or less is inadequate to predict a satisfactory response to treatment in the medium term. We propose a therapy interval of 4 weeks as a feasible period in order to judge the long-term efficacy of a ventilation mode. Further studies are needed to address this issue.

Despite the fact that the two ventilation modes compared were accurately matched for rate and volume during the daytime, a group of patients did not respond to $\mathrm{PCV}$. Thus, the adequate mechanical ventilation of a conscious and cooperative patient during the day does not always seem to be relevant to the nocturnal situation of a sleeping ventilated patient. The patients were adapted to the ventilator setting either in a sitting or a semirecumbent posture during the day. One reason for not responding to $\mathrm{PCV}$ may be the postural change of lung and chest wall mechanics during nocturnal ventilation. Compliance of the lung and chest wall were not assessed in this study. Furthermore, this study did not attempt to examine the electromyographic (EMG) activity of respiratory muscles, the sleep architecture, synchronicity between patient and ventilator, or leakages of the masks during nocturnal NMV. Analysis of these aspects would have permitted a deeper comprehension of the study outcome.

One may still criticize the decision to study the two controlled modes, without a triggered mode, in patients who have drive to breathe, as documented by $P 0.1$ measurements. Our reasoning was that since drive to breathe is normally reduced during sleep we were doubtful that adequate daytime $P 0.1$ would translate to fully effective triggering during sleep. Interesting parallels may be observed in COPD, where drive to breathe is usually high; however ElliotT et al. [15] demonstrated, in patients with COPD, that the facility to trigger was hardly used and that most patients used their ventilators in the controlled mode.

Since very few patients were nonresponders to IPPV, it could be argued that PCV is unnecessary. However, there are some features of PCV which make it attractive if it is effective. Thus, air leakage from the mask during sleep is not compensated for with IPPV, in contrast to PCV. We found other disadvantages to be alarm-associated noise and weight of the IPPV units used. However, the scores of disturbances refer exclusively to the specific devices tested in this study and not to the modality of ventilation as such. The disadvantages of IPPV, associated with practicability and costs, could change in the future with improvement in the design of the units. Finally, compared to PCV the IPPV units currently available in Germany are still more expensive.

We conclude that after initial treatment with intermittent positive pressure ventilation, pressure-controlled ventilation can stabilize the majority of patients suffering from chronic respiratory failure. In the subgroup of nonresponders, the efficacy of pressure-cycled ventilation was less than that of intermittent positive pressure ventilation, at equal settings. A period of 1 month was shown to be adequate for judging the medium-term outcome of a specific ventilation mode for noninvasive mechanical ventilation. If pressure-cycled ventilation shows the same efficacy as the preceding intermittent positive pressure ventilation, it can be assumed that pressure-cycled ventilation is an adequate alternative to intermittent positive pressure ventilation on a long-term 
basis. As a result of the present findings, a trial of pressure-cycled ventilation is thus recommended because it is more comfortable to use and less expensive.

Acknowledgements: The authors wish to thank M. Polkey and G. Laier-Groeneveld for thoughtful discussion, and B. Fischer for invaluable secretarial assistance.

\section{References}

1. Goldstone J, Moxham J. Weaning from mechanical ventilation. Thorax 1991; 46: 56-62.

2. Meyer TJ, Hill SH. Noninvasive positive pressure ventilation to treat respiratory failure. Ann Intern Med 1994; 120: 760-770.

3. Simonds AK, Elliott MW. Outcome of domiciliary nasal intermittent positive pressure ventilation in restrictive and obstructive disorders. Thorax 1995; 50: 604-609.

4. Brochard L, Mancebo J, Wysocki M, et al. Noninvasive ventilation for acute exacerbations of chronic obstructive pulmonary disease. $N$ Engl J Med 1995; 333: 817822.

5. Hill NS, Eveloff SE, Carlisle CC, Goff SG. Efficacy of nocturnal nasal ventilation in patients with restrictive thoracic disease. Am Rev Respir Dis 1992; 145: 365-371.

6. Renston JP, DiMarco AF, Supinski GS. Respiratory muscle rest using nasal BiPAP ventilation in patients with stable severe COPD. Chest 1994; 105: 1053-1060.
7. Restrick LJ, Fox NC, Braid G, et al. Comparison of nasal pressure support ventilation with nasal intermittent positive pressure ventilation in patients with nocturnal hypoventilation. Eur Respir J 1993; 6: 364370 .

8. Meecham Jones DJ, Wedzicha JA. Comparison of pressure and volume preset nasal ventilator systems in stable chronic respiratory failure. Eur Respir J 1993; 6: 1060-1064.

9. Quanjer PH, Dalhuijsen A, Van Zomeren BC. Summary equations of reference values. Bull Eur Physiopathol Respir 1983; 19 (Suppl. 5): 45-51.

10. Whitelaw WA, Derenne JP, Milic-Emili J. Occlusion pressure as a measure of respiratory center output in conscious man. Respir Physiol 1975; 23: 181-199.

11. Black LF, Hyatt RE. Maximal respiratory pressures: normal values and relationship to age and sex. Am Rev Respir Dis 1969; 99: 696-702.

12. Borg G. Perceived exertion as an indicator of somatic stress. Scand J Rehabil Med 1970; 2: 92-98.

13. Sachs L. Angewandte Statistik. Springer Verlag 1992; p. 649.

14. Vitacca M, Rubini F, Foglio K, et al. Noninvasive modalities of positive pressure ventilation improve the outcome of acute exacerbation in COLD patients. Intensive Care Med 1993; 19: 450-455.

15. Elliott MW, Mulvey DA, Moxham J, Green M, Branthwaite MA. Inspiratory muscle effort during nasal intermittent positive pressure ventilation in patients with chronic obstructive airways disease. Anaesthesia 1993; 48: 8-13. 